\title{
ANALYTIC FUNCTIONS THAT TAKE VALUES IN A CONVEX REGION
}

\author{
A. W. GOODMAN
}

1. Introduction. In this note the formulation of the problem takes much more space than the solution. In fact the proof of our only theorem is trivial, but the theorem itself seems to be interesting enough to justify this presentation.

We recall that in two papers Carathéodory $[1 ; 2]$ investigated power series.

$$
f(z)=1+\sum_{n=1}^{\infty} a_{n} z^{n}
$$

that are convergent in $E$, the unit circle $|z|<1$, and for which $\Re f(z)$ $>0$ in $E$. We let $\mathscr{F}(1, H)$ denote the family of such functions. In this notation $1=f(0)$, and $H$ denotes the half-plane $\Re w>0$, in which the values of $f(z)$ are required to lie for $z$ in $E$.

Herglotz [3] and later others (see Nevanlinna [6, p. 185]) proved that if $f(z) \in \mathcal{F}(1, H)$ then it has a representation as a Stieltjes integral, i.e.

$$
f(z)=\int_{0}^{2 \pi} \frac{1+e^{i t z}}{1-e^{i t z}} d \mu(t), \quad z \in E,
$$

where $\mu(t)$ is a nondecreasing function in $[0,2 \pi]$ for which

$$
\int_{0}^{2 \pi} d \mu(t)=1
$$

Conversely, it is obvious that any function defined by (2) and (3) belongs to the class $\mathcal{F}(1, H)$. If we observe that $F(z)=(1+z) /(1-z)$ maps $E$ univalently onto $H$, then (2), which has the form

$$
f(z)=\int_{0}^{2 \pi} F\left(e^{i t z}\right) d \mu(t), \quad z \in E,
$$

now appears as a weighted average of univalent functions, where the total weight, given by (3), is one.

We now invert this question, and ask what type regions $R$ have this property. Thus, suppose that $w=F(z)$ maps $E$ univalently onto $R$, and suppose that $f(z)$ is regular in $E, f(0)=F(0)$, and for $z \in E$, we have $f(z) \in R$. Does it follow that $f(z)$ has a representation of the

Received by the editors February 1, 1962. 
form (4), and conversely does each $f(z)$ defined by (4) have these properties? We shall prove that essentially the only region with this property is the half-plane.

2. Notation. Henceforth we let $R$ be a fixed region in the $w$-plane, and let $A_{0}$ be a fixed interior point of $R$. Let

$$
F(z)=A_{0}+\sum_{n=1}^{\infty} A_{n} z^{n}
$$

be regular and univalent in $E$ and map $E$ onto $R$. The function $F(z)$ is thus uniquely determined except for a rotation factor, but the form of equation (4) indicates that this factor is of no importance.

Let $F\left(A_{0}, R\right)$ be the class of all functions

$$
f(z)=A_{0}+\sum_{n=1}^{\infty} a_{n} z^{n}
$$

regular in $E$ and such that for all $z$ in $E, f(z)$ lies in $R$.

Finally let $\mathrm{S}\left(A_{0}, R\right)$ be the class of all functions generated by the Stieltjes integral (4), where $F(z)$ is the univalent function defined in equation (5), and $\mu(t)$ is any nondecreasing function that satisfies '(3). Accurately stated, the problem is to determine those pairs $\left(A_{0}, R\right)$ for which the two classes are the same, i.e.

$$
\mathcal{S}\left(A_{0}, R\right)=\mathscr{F}\left(A_{0}, R\right) .
$$

3. Properties of $R$. It is almost obvious that if (7) holds, then $R$ must be convex. For suppose that $R$ is not convex. Then there are two points $w_{1}, w_{2}$ in $R$ such that some point $w_{3}$ on the line segment joining $w_{1}$ and $w_{2}$ lies outside of $R$ or on the boundary. Let $w_{1}=F\left(z_{1}\right)$ and $w_{2}=F\left(z_{2}\right), z_{1}, z_{2}, \in E$. Since the line segment joining $w_{1}$ and $w_{2}$ meets the boundary of $R$ it is clear that we can (by shifting $w_{1}$ and $w_{2}$ along this segment if necessary) select them so that $\left|z_{1}\right|=\left|z_{2}\right|$, i.e. $z_{2}=z_{1} e^{i \alpha}, \alpha$ real. Let $w_{3}=\mu_{1} w_{1}+\mu_{2} w_{2}$ where $\mu_{1}+\mu_{2}=1$ and $\mu_{1}, \mu_{2}>0$. Then the function

$$
f(z)=\mu_{1} F(z)+\mu_{2} F\left(e^{i \alpha_{z}}\right)
$$

is in the class $\delta\left(A_{0}, R\right)$. But for $f(z)$ given by $(8), f\left(z_{1}\right)=\mu_{1} F\left(z_{1}\right)$ $+\mu_{2} F\left(z_{2}\right)=w_{3} \notin R$. Hence $f(z) \notin \mathscr{F}\left(A_{0}, R\right)$. Consequently, if (7) holds then $R$ must be convex.

We assume henceforth that $R$ is convex, and an obvious consequence of this convexity is the inclusion relation

$$
\mathcal{S}\left(A_{0}, R\right) \subset \mathcal{F}\left(A_{0}, R\right) .
$$


Finally we remark that (7) is invariant under rotations, magnifications, and translations, as long as the transformation acts on both the constant $A_{0}$ and the region $R$. Thus if (7) is satisfied and $b$ and $c \neq 0$ are any complex constants, then

$$
\mathcal{S}\left(A_{0}-b, R-b\right)=\mathcal{F}\left(A_{0}-b, R-b\right)
$$

and

$$
S\left(c A_{0}, c R\right)=\mathcal{F}\left(c A_{0}, c R\right) \text {. }
$$

The proof is simple, and hence we omit it. However, in view of (9), it is only necessary to prove the opposite inclusion to obtain (10) or (11).

4. The theorem. Using (10) and (11) we can now normalize the problem by taking $A_{0}=0$. Further we can select $c$ in (11) so that the univalent function $F(z)$ has the form

$$
F(z)=z+\sum_{n=2}^{\infty} A_{n} z^{n}
$$

Since $R$ is a convex, it follows from Loewner's Theorem on convex regions $[4$, p. $103 ; 5$, p. 223$]$ that $\left|A_{n}\right| \leqq 1$ for $n=1,2,3, \cdots$ with equality if and only if

$$
F(z)=\frac{z}{1-e^{i \beta} z}=\sum_{n=0}^{\infty} e^{i n \beta} z^{n+1}, \quad(\beta, \text { real }) .
$$

Let $f(z) \in S(0, R)$. Then from (4) and (12)

$$
\begin{aligned}
f(z) & =\int_{0}^{2 \pi} F\left(e^{i t} z\right) d \mu(t)=\int_{0}^{2 \pi}\left[e^{i t} z+\sum_{n=2}^{\infty} A_{n} e^{i n t} z^{n}\right] d \mu(t) \\
& =a_{1} z+a_{2} z^{2}+\cdots
\end{aligned}
$$

and consequently for $n=2,3,4, \cdots$

$$
\left|a_{n}\right| \leqq \int_{0}^{2 \pi}\left|A_{n} e^{i n t}\right| d \mu(t)=\left|A_{n}\right| .
$$

On the other hand for each positive integer $n, f(z) \equiv F\left(z^{n}\right)=z^{n}$ $+\cdots \in \mathcal{F}(0, R)$. If now $S(0, R)=\mathcal{F}(0, R)$ then by (14) applied to the function $F\left(z^{n}\right)$ we have

$$
1 \leqq\left|A_{n}\right| \quad n=2,3,4, \cdots
$$

Combining this with the previous remark that $\left|A_{n}\right| \leqq 1$ for convex regions shows that $\left|A_{n}\right|=1$ for each $n$. Consequently $F(z)$ has the form (13). This proves the first half of 
Theorem 1. If $\mathcal{S}\left(A_{0}, R\right)=\mathfrak{F}\left(A_{0}, R\right)$ then $R$ is a half-plane. Conversely, given any half-plane $R$ and any point $A_{0} \in R$, we have $\mathrm{S}\left(A_{0}, R\right)$ $=\mathfrak{F}\left(A_{0}, R\right)$.

The proof of the converse part is immediate using (10) and (11).

5. Some open questions. It is quite natural to try to obtain the identity of the two classes $S$ and $\mathcal{F}$ for convex regions other than the half-plane by altering the definition of $S$ so as to include more functions. Suppose that just as before $F(z)$, given by (5) maps $E$ univalently onto $R$. Then the function $F\left(e^{i t}\left(z+\rho e^{i \alpha}\right) /\left(1+z \rho e^{-i \alpha}\right)\right)$ does the same, and so a weighted average of such functions will be in $\mathcal{F}\left(A_{0}, R\right)$ if the normalization is selected properly. Let $\mathrm{S}_{3}\left(A_{0}, R\right)$ be the class of functions $f(z)$ generated by the triple Stieltjes integral

$$
f(z)=\int_{0}^{2 \pi} \int_{0}^{2 \pi} \int_{0}^{1} F\left(e^{i t} \frac{z+\rho e^{i \alpha}}{1+z \rho e^{-i \alpha}}\right) d \mu(\rho, \alpha, t)
$$

where $F(z)$ is fixed and $\mu(\rho, \alpha, t)$ is a nondecreasing function over the three-dimensional set $0 \leqq \rho \leqq 1,0 \leqq \alpha \leqq 2 \pi, 0 \leqq t \leqq 2 \pi$, subject to the normalizing conditions

$$
\begin{aligned}
\int_{0}^{2 \pi} \int_{0}^{2 \pi} \int_{0}^{1} d \mu(\rho, \alpha, t) & =1 \\
\int_{0}^{2 \pi} \int_{0}^{2 \pi} \int_{0}^{1} F\left(\rho e^{i(\alpha+t)}\right) d \mu(\rho, \alpha, t) & =A_{0} .
\end{aligned}
$$

Then, we ask, for what pairs $A_{0}, R$ do we have $S_{3}\left(A_{0}, R\right)=\mathscr{F}\left(A_{0}, R\right)$ ? Of course, it is clear that, just as before, the region $R$ must be convex.

More generally, we may take any kernel $F\left(z, t_{1}, t_{2}, \cdots, t_{n}\right)$ analytic in the complex variable $z$ for $|z|<1$, and continuous in $t_{1}, t_{2}, \cdots, t_{n}$ and let $S(F)$ be the class of functions $f(z)$ generated by the multiple Stieltjes integral

$$
f(z)=\int_{0}^{1} \cdots \int_{0}^{1} F\left(z, t_{1}, t_{2}, \cdots, t_{n}\right) d \mu\left(t_{1}, t_{2}, \cdots, t_{n}\right)
$$

with suitable normalization. Under what conditions on the kernel $F$ do we have $\mathrm{S}(F)=\mathfrak{F}\left(A_{0}, R\right)$ ? In this situation the region $R$ is the convex cover of the set of all values of $f(z)$ as $f$ runs through $S(F)$.

\section{REFERENCES}

1. C. Carath€odory, Über den Variabilitätsbereich der Koeffizienten von Potensreihen die gegebene Werte nicht annehmen, Math. Ann. 64 (1907), 95-115. 
2. - Über den Variabilitätsbereich der Fourier'schen Konstanten von positiven harmonischen Funktionen, Rend. Circ. Mat. Palermo 32 (1911), 193-217.

3. G. Herglotz, Über Potenzreihen mit positivem reellen Teil im Einheitskreis, Ber. Sachs. Akad. Wiss. Leipzig Math.-Phys. K1. 63 (1911), 501-511.

4. C. Loewner, Üntersuchungen über die Verzerrung bei Konformen Abbildungen des Einheitskreises $|z|<1$ die durch Funktionen mit nicht verschwindender Ableitung geliefert werden, Ber. Sachs. Akad. Wiss. Leipzig, Math.-Phys. Kl. 69 (1917), 89-106.

5. Z. Nehari, Conformal mapping, McGraw-Hill, New York, 1952.

6. R. Nevanlinna, Eindeutige Analytische Funktionen, J. W. Edwards, Ann Arbor, Michigan, 1944.

UNIVERSITY OF KENTUCKY

\section{VANISHING CENTRAL DIFFERENCES}

RICHARD F. DEMAR

Given a sequence $\left\{a_{n}\right\}_{n=0}^{\infty}$ of complex numbers, a number of theorems have been proved concerning implications of the vanishing of certain differences $\Delta^{n} a_{0}$ if the given sequence satisfies some growth restriction. The first such result, proved by Agnew [1], states that if $\left\{a_{n}\right\}$ is bounded and $\Delta^{2 n} a_{0}=0$ for all $n$, then $a_{n}=0$ for all $n$. If all the odd differences are zero, the sequence is constant. Fuchs [6] proved the following: Let $a_{n}=o\left(n^{k}\right)$ for some positive number $k$, and let $n_{j}$ be a subsequence of the positive integers such that if $n(R)$ is the number of $n_{j}<R$, then $n(R) \geqq R / 2$ for $R>R_{0}$. If $\Delta_{j}^{n} a_{0}=0$ for all $n_{j}$, then $a_{n}=p(n)$ where $p(x)$ is a polynomial of degree less than $k$. Buck [4] assumed only that lim sup $\left|a_{n}\right|^{1 / n}<1$ and $\Delta^{n} a_{0}=0$ for all $n$ belonging to a set of positive integers of density $d>\frac{1}{3}$ and proved there is a function $f$ of exponential type whose growth function $h(\theta)$ satisfies $h( \pm \pi / 2)<\pi$ such that $f(n)=a_{n}$ for all $n$. In this paper, we show that if the given sequence is extended to $\left\{a_{n}\right\}_{n=-\infty}^{\infty}$ by letting $a_{-n}=a_{n}$, then the vanishing of certain of the even central differences $\Delta^{2 n} a_{-n}$ has similar implications. Or, letting $a_{-n}=-a_{n-1}$, vanishing of odd differences $\Delta^{2 n-1} b_{-n}$ gives similar results.

If $G$ is a connected set, let $K[G]$ denote the class of all entire functions of exponential type whose conjugate indicator diagrams $D(f)$ are contained in $G$. If $G$ is the rectangle $\{x+i y|| x|\leqq a ;| y \mid \leqq c\}$, then $K[a, c]$ will be used for $K[G]$. Let $C_{z, n}$ denote the polynomial $z(z-1) \cdots(z-n+1) / n !$

Certain results concerning the sequence $\left\{\mathscr{L}_{n}\right\}$ of Stirling functionals given by $\mathscr{L}_{n}(f)=\Delta^{n} f(-n / 2)$ will be needed. These functionals

Presented to the Society, January 24, 1961, under the title, A theorem on vanishing differences; received by the editors November 24,1961 and, in revised form, December $12,1961$. 